\title{
Relaciones entre escuela y familias inmigrantes. Estudio de un caso $^{1}$
}

\section{Relationships between schools and immigrant families. A Case Study}

\author{
Antonio Bautista García-Vera ${ }^{2}$ \\ bautista@edu.ucm.es \\ ENCARNACIÓN ARACIL RODRÍGUEZ \\ enaracil@cps.ucm.es \\ Mercedes Blasco Torrejón \\ cherche@telefonica.net \\ María Caballero Hernández Pizarro \\ mariachp@edu.ucm.es \\ Jorge Cujó Arenas \\ jorgek@edu.ucm.es \\ Universidad Complutense de Madrid, España
}

\section{Resumen:}

Este artículo versa sobre la participación de padres inmigrantes en los colegios a los que asisten sus hijos. Su valor o aportación reside en incluir la narración audiovisual en la relación entre la escuela y la comunidad. Exponemos los primeros resultados del proyecto que investiga la naturaleza de las relaciones entre un grupo de estas familias con la institución educativa, promovidas por narraciones verbales y fotográficas grupales, iniciadas y apoyadas por reuniones en las aulas del colegio y continuadas en sus contextos familiar y social. Durante el curso 2011/12, trabajamos con las familias de un grupo de $4^{\circ}$ de EP del

\begin{abstract}
:
This article focuses on the participation of immigrant parents in the schools attended by their children. Its main contribution lies in the fact that audiovisual narratives have been used for portraying the relationship between school and community. We present the first results of the project which investigates the nature of the relationships between a group of these families with the educational institution, promoted by verbal and photographic narratives group meetings initiated and supported in school classrooms and continued in their family and social contexts.

During the school year 2011/12, we
\end{abstract}

1 Proyecto subvencionado por el Ministerio de Economía y Competitividad, referencia EDU2011-23380

2 Dirección para correspondencia (correspondence address):

Antonio Bautista García-Vera. Dpto. de Didáctica y Organización Escolar. Facultad de Educación. Universidad Complutense de Madrid. Rector Royo Vilanova s/n, Ciudad Universitaria, Edificio La Almudena. 28040, Madrid (España). 
CEIP Jaime Vera de Madrid, España, que tiene un $89 \%$ de estudiantes inmigrantes. Proporcionamos reuniones de discusión sobre las historias que pretendían narrar. Además de la observación y del registro en audio de sus manifestaciones espontáneas en las sesiones de narración, se hicieron entrevistas informales a las familias participantes para dar validez al estudio. Las familias dieron su aprobación para que dichas sesiones fueran grabadas.

Entre las conclusiones de este estudio cabe señalar que la influencia del director y de la tutora ha sido fundamental en la participación de las familias. Hemos comprobado que hay contraste entre el conocimiento superficial que manifestaron tener unos padres de otros al principio del año escolar, y la relación más estrecha que existió entre ellos al final. Hay que mencionar el papel relevante que han tenido las preguntas y cuestiones realizadas sobre el contenido narrado para mejorar el conocimiento mutuo de los participantes.

\section{Palabras clave:}

Relación familia-escuela, educación de inmigrantes, comunicación intercultural, narración. worked with families of a group of 4 th Primary Education students in the Jaime Vera School in Madrid, Spain. Among the pupils enrolled, $89 \%$ share an immigrant background. Meetings provide discussion intended to narrate the stories. In addition to observation and audio recording of its manifestations in spontaneous storytelling sessions, informal interviews with participating families were carried out to validate the study. Families gave their approval for these sessions to be recorded.

Among the conclusions of this study it should be noted that both the school's director and the tutor played a determinant role in engaging families to participate. We have found that there is a noticeable contrast between the superficial knowledge that parents have expressed about each other at the beginning of the school year, and the closer relationship that existed among them at the end of that period. Finally, the positive impact of the questions asked during the participants' narratives on their mutual understanding should be highlighted.

\section{Keywords:}

Family-school relationship; immigrant education; intercultural communication; storytelling.

\section{Résumé:}

Cet article aborde la participation des parents immigrants dans les écoles où leurs enfants étudient. La valeur de son apport réside dans l'inclusion des narrations audiovisuelles comme une technique positive de communication entre l'école, la sphère familiale et la communauté. Nous présentons ici les résultats préliminaires d'un projet qui étudie la nature des relations entre un groupe de ces familles avec l'institution éducative. À tel effet, on a promu des narrations verbales et photographiques initiées et appuyées par des réunions dans les salles de classe de l'école et continués plus tard dans le contexte familial et social.

Pendant I'année scolaire 2011/12, nous avons travaillé avec les familles d'un groupe appartenant à la quatrième année de l'enseignement primaire à l'école publique Jaime Vera de Madrid, en Espagne. Cette école dénombre un pourcentage élevé des enfants immigrants, représentés par le $89 \%$ sur le total des scolarisés.

Nous avons proposé des réunions pour que ces familles discutent sur les histoires qu'ils voulaient raconter. En plus de l'observation et du registre audio des manifestations spontanées pendant les séances narratives, nous avons réalisé des entretiens informels avec les familles participantes pour pouvoir valider notre étude. Les familles ont exprimé leur accord pour l'enregistrement digital des sessions.

Parmi les conclusions de cette étude il faut remarquer le rôle fondamentale joué par le directeur et la tutrice pour encourager la participation des familles. Nous avons trouvé des différences entre la connaissance superficielle des autres familles que les parents ont 
exprimé au début de l'année scolaire, et la relation étroite qui existait entre eux à la fin de l'année scolaire. Finalement, il faut signaler le rôle des questions posées sur le contenu des narrations pour accroître la connaissance mutuelle des participants.

\section{Mots clés:}

Relation famille-école; l'éducation des immigrants; la communication interculturelle; la narration.

Fecha de recepción: 18-7-14

Fecha de aceptación: 26-2-2015

\section{Introducción}

Las relaciones entre escuela y familia no han sido fáciles y se han caracterizado por ser distantes, adversas y de desvalorización mutua (Collet y Tort, 2008; Delgado, 2008), o como la crónica de un desencuentro (Fernández Enguita, 1993). La desconexión e incomunicación entre ambas no han contribuido en la mejora del desarrollo personal y social de los humanos implicados en la educación, porque, por ejemplo, la desconfianza en el profesorado no ha ayudado a su desarrollo profesional, y la ausencia de la participación de padres en el centro ha sido un obstáculo en la formación ciudadana del alumnado (Banks, 2004; Parker, 2003).

Ante esta situación preocupante, y apoyándonos en los datos que hemos obtenido en el trabajo realizado durante el año 2012 en el CEIP Jaime Vera de Madrid, en este artículo abordamos las relaciones entre la escuela y familias inmigrantes con diferente nivel de conocimiento del castellano. Contribuimos, por lo tanto, en la construcción del conocimiento que, además de reducir la tasa de fracaso escolar de este alumnado extranjero, ayude a incorporar a sus padres y madres a la comunidad y país de acogida; pues, entre otros argumentos, el Consejo de Europa (2007) recomendó una formación tecnológica de los humanos como elemento necesario para una educación ciudadana; $y$, en un dictamen posterior (Recomendación de la CE del 20 de agosto de 2009), se aprobó la Iniciativa i2010 que considera la formación mediática como elemento básico en la construcción de una sociedad de la información y comunicación más incluyente. 


\section{Revisión del estado de la cuestión}

La relación entre familias inmigrantes y centros educativos tiene múltiples aristas y, consecuentemente, existen bastantes indagaciones sobre ellas. Una se refiere a la participación de las familias en la escuela a la que asisten sus hijos, valorándose la misma a través del rendimiento académico de estos. Una conclusión compartida por distintos autores consultados es la escasa participación de los padres en general y de los inmigrantes en particular, lo poco que se implican en el funcionamiento del colegio y la dificultad que tal ausencia supone en el éxito escolar de sus hijos (Essomba, 2006; García Castaño, Rubio y Bouachra, 2008; Cabello y Gradaílle, 2008; Chamberlin-Quinlisk, 2010).

La indagación en las estrategias de relación utilizadas por los colegios para comunicarse y colaborar con las familias ha conformado otro grupo de estudios (McCaleb, 1997; Castelli, Mendel y Ravn, 2003; Diez y Terrón, 2006; Henderson, Mapp, Johnson y Davis, 2007; Parcerisa, 2008; Martínez, Rodríguez y Gimeno, 2010). Aunque los consejos escolares y las asociaciones de madres y padres de alumnos son los órganos institucionales que vehiculan dicha relación usando las entrevistas, reuniones y correspondencia escrita, se han experimentado otras iniciativas para mejorarla, tales como plantear ámbitos de ayuda de los padres la institución (por ejemplo, la creación y mantenimiento de una página web, blog,.. o la colaboración en las excursiones y salidas de sus hijos del colegio), o solicitar mayor implicación en la elaboración del proyecto educativo del centro y en la gestión del día a día del mismo. En estos trabajos aparece tímidamente la presencia de herramientas tecnológicas para concretar la reunión de los padres con el profesorado, siendo la agenda escolar y las anotaciones textuales las más frecuentes, y el teléfono y el correo electrónico los menos utilizados.

¿Cómo se contempla la inmigración y los inmigrantes en su relación con el contexto escolar y social donde viven? es otro de los temas de estudio. Se ha abordado desde varios puntos de vista: psicológico, antropológico, sociológico, etcétera. Así, sobre los mecanismos de inclusión y exclusión de estos colectivos en las comunidades y países de acogida (Ogbu, 1993; Franzé, 2008), Ogbu planteó que para entender las relaciones interculturales había que estudiar las fuerzas comunitarias y el efecto de estas sobre el comportamiento social y escolar del alumnado minoritario. Algo parecido se observa en los trabajos donde los elementos del 
curriculum han mediado la participación de las familias inmigrantes en el centro educativo, centrándose en dimensiones como la selección de contenidos y la organización espacio-temporal del alumnado diverso (Bolivar, 2006; Johansson, 2009; Torres, 2010).

Fue así como en la investigación realizada abordamos algunas de las ausencias en los estudios revisados anteriormente, concretamente, el uso de narraciones audiovisuales para fomentar la relación entre las familias inmigrantes y el colegio al que asisten sus hijas e hijos.

\section{Objetivos de la investigación}

Ante el anterior estado de la cuestión, abordamos los siguientes objetivos de conocimiento:

1) Conocer si la historia elaborada por un padre o madre suscita la participación de los otros contertulios. De ser así, indagar si ayudan a un padre los comentarios y sugerencias de otros sobre la narración que ha expuesto, para profundizar y conocer mejor los contenidos de la misma.

2) Saber en qué medida han influido el equipo directivo del centro y la tutora de sus hijos en el desarrollo de las relaciones de los padres con el centro.

3) Finalmente, al final del curso escolar, conocer si ha cambiado el significado que tiene el colegio para los padres participantes. Es decir, saber si el próximo curso irán a la escuela para ver y reunirse con otros padres y maestros, o solo para hablar con los profesores sobre el progreso de sus hijos.

\section{Descripción de la recogida de datos}

En el mes de octubre de 2011 presentamos el proyecto al equipo directivo y al claustro de profesores del CEIP Jaime Vera de Madrid (España), centro que tiene un $89 \%$ de estudiantes inmigrantes. Valoraron bien la propuesta de trabajo y acordaron participar en su desarrollo. La profesora-tutora de un grupo de $4^{\circ}$ curso de Educación Primaria (niños y niñas de 10 años) nos abrió las puertas de su aula, nos puso en comunicación e invitó a las 19 familias de su alumnado a una reunión en el colegio. 
A la primera reunión asistieron 14 padres y madres; de estas, 6 dejaron de asistir por distintas razones. Desde entonces, y hasta final de año, mantuvieron su continuidad en las reuniones quincenales 2 familias de República Dominicana, 1 de Marruecos, 1 de Camerún, 1 de Bangladesh, 1 de Perú, 1 de Ecuador y 1 de España. Estas sesiones o encuentros se desarrollaron los viernes a las 16 horas, momento en el que todos podían asistir.

\subsection{Proceso de recogida y análisis de datos}

Procedimos de la siguiente forma:

- Hicimos un análisis previo mediante conversaciones informales para descubrir cuánto sabían las familias unas de otras. Como expondremos en los datos, las familias tenían un conocimiento superficial entre ellas, aunque sus hijos habían permanecido varios cursos en ese colegio.

- Generamos situaciones o reuniones de discusión en torno a la presentación de relatos proporcionadas por los padres (creados por ellos, o seleccionados de fuentes documentales) sobre las historias que pretendían narrar. De esta forma, la narración o relato de historias es uno de los elementos claves de este estudio, por permitir conocer cómo experimentan o viven esas relaciones padres y profesores (Clandinin y Connelly, 2000; Mackernan, 2008; Flick, 2012; Gibbs, 2012; entre otros). Pues como dice Gibbs (2012, p. 85) «Las expresiones y el vocabulario y las metáforas compartidas pueden decirnos mucho sobre el modo en que los grupos sociales se ven a sí mismos y cómo explican sus experiencias».

- Además de la observación y del registro en audio de sus manifestaciones espontáneas en las sesiones de narración, se hicieron entrevistas informales a los padres y madres participantes para aclarar o resolver las posibles dudas que surgieron en el proceso de transcripción y análsis del contenido de dichas relaciones.

- Describimos el proceso seguido a través de este proyecto sobre la evolución de la relación de los padres con la escuela y viceversa; para ello, utilizamos datos recogidos, transcritos y validados de dichas reuniones.

- La validación se hizo por triangulación de los puntos de vista de tres padres (Said, Mar y Eliot), la profesora-tutora y dos miembros 
del equipo de investigación, mediante la lectura y las correspondientes modificaciones de los informes sobre el análisis y descripción señaladas anteriormente, elaborados por el coordinador y el resto de colaborores del grupo.

- Se elaboraron categorías comprensivas de ese proceso, que presentamos a continuación. Para tal fin, seguimos los pasos propuestos por Gibbs (2012), es dicir, en primer lugar realizamos una codificación temática «guiada por los datos» de las conversaciones en audio transcritas y, posteriormente, procedimos a la categorización sobre la codificación previa efectuada.

- Las familias participantes dieron su aprobación para que fuese grabado el contenido de las sesiones de narración. Por nuestra parte, manifestamos el compromiso de usarlo solo para fines docentes y de investigación.

\section{Presentación y análisis de datos}

\subsection{Sobre la situación de partida}

Las entrevistas iniciales que, a modo de conversaciones informales, mantuvimos con los padres asistentes, unidas a las observaciones y registros auditivos realizados en las primeras sesiones de narración, nos permiten afirmar que los padres y madres, aún llevando una relación de 3 y 4 años con el colegio, tenían un conocimiento superficial del resto de los progenitores de los compañeros de sus hijos e hijas; pues como dijo Mar (madre de Enrique): "conozco de vista a otros padres de cuando la profesora hizo una reunión de grupo al principio del año y, luego, de vez en cuando, los he visto en la puerta del colegio», (entrevista del día 22/11/2011); o Rocío (madre de Lisbeth) «Yo conozco a poquitas mamás y papás, porque trabajo de cocinera y no puedo llegar a las reuniones del colegio que no sean los viernes... Las que conozco son porque me lo dice mi hija cuando las veo por la calle o en el parque» (reunión 2/12/2011); o la manifestación que hizo Raudi (madre de Rashell) al valorar las sesiones de narración «Me parecen muy interesantes, y muy buena idea, porque así realmente podemos conocer no solamente a los niños que están con sus compañeritos, sino que también podemos conocer los padres de los amigos de nuestros hijos» (entrevista 2/12/2011). 


\subsection{Sobre las situaciones de narración desarrolladas en las reuniones con padres y madres en el colegio}

Analizando las transcripciones de las reuniones del año 2012, encontramos que las situaciones más frecuentes que encontramos fueron las que hemos denominado descriptivas. En ellas, las relaciones se caracterizaron porque los protagonistas de las historias narradas manifestaron los propósitos de las mismas, y no hubo momentos en los que fuese necesario explicitar las intenciones y motivaciones de quienes tenían la palabra. Entendemos que esa es una razón importante que explica el porqué no emergieron interpretaciones ni preguntas sobre lo expuesto por los padres y madres cuando intervinieron para comunicar el tema o evento que consideraron relevante sobre su país.

Veamos algunos fragmentos que ilustran este tipo de situaciones; por ejemplo, cuando Eliot tomó la palabra en una de las primeras reuniones del grupo:

«Eliot: Nosotros somos de Perú y vamos a hablar de comidas típicas y bailes típicos.

Piedad: ¿De qué zona de Perú?

Eliot: Somos de la Sierra. Perú se divide en tres regiones naturales: costa, sierra y selva. Como voy a hablar de la comida y de sus bailes, primero voy a hablar de la costa. Al tener mar y estar pegado a la playa, las comidas son las siguientes... "ceviche" de pescado, que se compone de pescado, limón y patata, que en mi país se le dice "papa". Al lado de la fotografía está. ¡Vale! Para ir abreviando, si te vas desde la costa hacia la tierra, en la serranía se toma mucho caldo, mucha sopa, ¿no sé si me entienden?

Antonio: O sea, hace más frío, se necesitan más calorías.

Eliot: Y se come más espeso, con boniato, que en mi país se dice "capote".

Pilar: ¿Cómo dices que llamas al boniato?

Eliot: Capote. Y en la selva, pues hay más plátanos, como está pegado al Ecuador y Colombia...por la selva de Perú se comen mucho los patacones, y los mangos, las papayas, frutas tropicales... Y los bailes típicos, en la costa hay uno que se llama la marinera norteña». (Reunión del 16/12/2011).

También, hemos observado que otra de las bondades de este tipo de situación descriptiva es ser un nuevo reto de indagación para quien ex- 
pone; por ejemplo, en la sesión donde Mar comunicó algunos detalles de la historia que estaba elaborando:

«Mar: Las corridas de toros son un espectáculo que consiste en llevar a un toro a pie o a caballo. Y en un recinto cerrado eso es una plaza de toros.

Mar: En las corridas solo puede participar como matador el torero que ha tomado la alternativa.

Eliot: ¿Qué diferencia hay entre un banderillero y un matador?

Mar: Pues que el matador es el torero que hace todo el recorrido, desde que sale el toro por el toril hasta que muere.

Chayo: $Y$ de hecho hay algunos toreros que ellos mismos ponen las banderillas.

Eliot: Dices que es a principios del s. XII pero ¿quién lo introduce en España?... porque estos toros que salen en las corridas son especiales, de lidia, de raza, ¿pero cómo se introduce ese toro? ¿Quién sabe si de Francia, o de dónde...?

Mar: Me ha pillado...

Eliot: Porque ahí pone el s. XII, pero habría que ir mucho más atrás». (Reunión del 10/02/2012)

Es evidente, que la pregunta de Eliot supuso para Mar un incentivo para seguir indagando sobre el origen del toro y de las corridas de toros en España; en este sentido, las preguntas sobre el contenido de algunas descripciones fueron básicas para iniciar un pensamiento reflexivo de cada participante consigo mismo y, de esta forma, dar respuesta a las dudas suscitadas.

\subsection{Sobre la influencia de la relación de los representantes de la escuela en estos eventos y, consecuentemente, en la participación de las familias en el proyecto}

Al hablar de los representantes del colegio nos referimos a las figuras del director y de la profesora tutora del alumnado de $4^{\circ}$ curso, grupo B, de Educación Primaria que ha intervenido. La apertura y disponibilidad del primero, José, ha sido excelente en todo momento, poniéndonos en comunicación con la profesora de esos estudiantes, Piedad, y permitién- 
donos el acceso al colegio en horario extraescolar. De igual forma, la tutora desde el primer día mostró su compromiso convocando a los padres por carta a una reunión, con el visto bueno del director, para tratar el punto "Presentación del Proyecto que realizarán las familias de esta clase en colaboración con la Facultad de Educación de la UCM", informando del propósito del mismo "el conocimiento de las familias entre sí cuando se hacen tareas comunes como buscar fotos, revistas, poesías, recetas, canciones, etcétera, que compondrán las historias que narrarán sobre su país", entregando una encuesta a cada familia, donde cada una manifestó por escrito sus días de preferencia o disponibilidad para las reuniones, y participando en las mismas que, como ya señalamos, fueron los viernes a las 16 horas en el mismo aula donde ella impartía clase.

En este sentido, la asistencia del director no fue continua a lo largo de todo el proyecto ni de todas las sesiones, lo hizo sobre todo en las primeras y de forma espontánea en algunas de las siguientes; por ejemplo en la visita que hizo en la reunión del día 2 de diciembre de 2011, donde manifestó:

"Es un gusto verlos por algo relacionado con sus hijos, pero diferente a lo que estamos acostumbrados, para matemáticas y otras materias. Nos quejamos de que no tenemos mucha respuesta de los padres, pero hoy estáis un grupo que es más numeroso de lo que normalmente asiste al colegio... a ver si se puede ir ampliando. Muchas gracias".

Su presencia ha sido necesaria y valiosa, pues ha dado legitimidad institucional al proyecto $y$, consecuentemente, ese respaldo ha contribuido en la buena respuesta mostrada por las familias. Esta colaboración del director se complementó con la participación permanente de la tutora, cuya presencia dio fundamento educativo a las acciones desarrolladas en el proyecto, pues ayudaba a dar confianza a las propuestas de los padres con indicaciones sobre nuevas posibilidades que abrían sus planes de acción; por ejemplo, ante una manifestación de Said (Bangladesh), Piedad dijo:

"Aunque aquí en España no exista monzón, está bien que tengamos alguna imagen explicando el monzón, ¿eh? porque eso va a gustar". (Reunión del 2/12/2012). 
O cuando Eliot (Perú) expuso algunos de los platos típicos, Piedad manifestó:

"Vamos a dar las recetas a todos y luego un baile con una música que cantemos todos y hacer algo... porque en el día de la presentación de todas las historias sobre vuestros países va haber niños y padres". (Reunión del 2/12/2012).

También, la profesora ha dado respaldo docente y ha sido puente de unión entre los padres y el aprendizaje, desarrollo y vida de sus hijos e hijas en el colegio; por ejemplo, en una de las reuniones dedicadas a narrar las historias que habían elaborado, Piedad hizo referencia a las fotografías aportadas por Said:

"Muy bien Said, has hecho una buena narración. Están muy bien las imágenes que has buscado y traído sobre las estaciones del año en Bangladesh, donde por ejemplo se ven las consecuencias de las inundaciones producidas en el monzón. Es una imagen que ha gustado y sorprendido a los niños cuando se la mostré" (Reunión 25/05/2012).

Esta participación de la profesora tutora ha sido determinante en el proyecto, ha sido básica para que se haya podido desarrollar, porque, aunque la presencia del director también fue necesaria, no fue suficiente por sí sola, tuvo efecto por la implicación de esta maestra para lograr el compromiso de los padres y madres. Unos datos que justifican esta valoración están relacionados con la respuesta de las familias en relación con la presencia de Piedad en el colegio para realizar este estudio.

\subsection{Sobre la respuesta de los padres}

La presencia de los padres y madres en el colegio no ha sido regular. Observando la figura 1, podemos manifestar que hubo dos puntos de inflexión en dicha asistencia: 


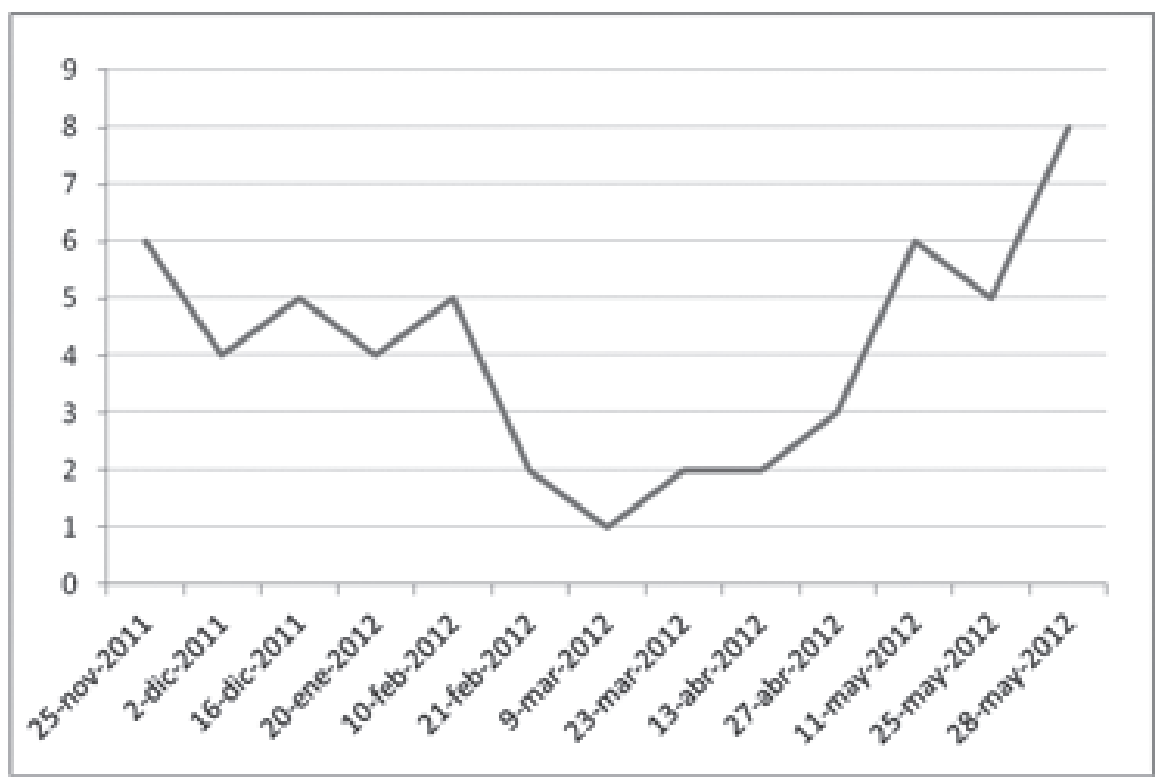

Figura 1. Número de familias asistentes a cada reunión.

El momento de inflexión 1 se produjo a partir de la quinta reunión, celebrada el 10/02/2012, cuando Piedad anunció que "durante un tiempo no asistiría a las sesiones por un asunto particular". Durante las cinco reuniones siguientes la presencia de las familias se redujo a menos de la mitad.

El momento de inflexión 2 tuvo lugar en la décima reunión, cuando se comunicó a los padres y madres "que la maestra se incorporaba para ir preparando la sesión final en la que se narrarían y exhibirían las historias que se habían venido elaborando durante estas semanas" (Antonio, 27/04/2012). En las tres reuniones que tuvieron lugar desde esa fecha la presencia de los padres se triplicó.

Sobre las motivaciones que llevaron a estas familias a no asistir durante las cinco sesiones transcurridas entre los dos momentos de inflexión, son interesantes las reflexiones aportadas por la única madre que asistió a la reunión del 9 de marzo de 2012. Ante la siguiente cuestión planteada por una profesora del grupo de investigación "ipor qué no habrán venido las familias que se comprometieron a asistir a las sesiones de narración?", manifestó : 
"Pienso que hay unos padres que no pueden venir porque les han cambiado el turno en su trabajo o que han tenido algún problema, es lógico que para ellos antes sea el trabajo que venir al colegio. He hablado con Raudi, Eliot, Said y vienen porque les gusta hablar de sus países y conocer el de otros, porque quieren saber más cosas de lo que hacen sus hijos en el colegio.. y, claro, si no viene Piedad, luchan menos por cambiar un turno de trabajo" (Mar. Reunión del 9/03/2012).

Posteriormente, completó su punto de vista con esta otra información:

"Pero otros padres que no han venido porque no viene Piedad, porque se apuntaron al proyecto para quedar bien con ella, para proteger a sus hijos; si Piedad no viene, no se entera de que ellos no vienen y sus hijos no pagan las consecuencias" (Mar, reunión del 9/03/2012).

Ante esta información tan reveladora de las motivaciones de algunas familias, aprovechamos los momentos del final de las tres últimas reuniones para mantener entrevistas informales con algunos padres para conocer cómo habían vivido las sesiones en las que habían participado:

"Una cosa que me atrajo como un imán fue poder hablar con la maestra cuando ella no era maestra pero seguía estando en la clase para que nosotros podamos conocernos más. Piedad contaba cosas de lo que hacía mi hijo y de cómo contaba lo feliz que era en casa cuando desarmábamos la compra, cosa que yo no sabía que eso le hiciera tan feliz" (Eliot, 25/05/2012)

"Yo veía a mi niñita contenta porque venía a las reuniones, porque luego Piedad les decía lo que hacíamos y lo bonito de mi país. Mi hijita estaba alegre y se sentía importante" (Rocio, 28/05/2012)

Comprobamos que en la respuesta de estos padres se volvió a poner de manifiesto la función docente y de unión de la tutora entre los padres y la vida de sus hijos en el colegio; función que consideramos ha sido esencial en el desarrollo del proyecto. 


\section{Conclusiones}

Los datos proporcionados anteriormente permiten responder a las cuestiones formuladas en este artículo.

1) Hemos comprobado que en todo el proceso de narración, se ha suscitado la participación del resto de los contertulios; aunque la frecuencia de la misma no ha sido igual en todos, porque ha habido casos como el de Manuel (Ecuador) que, aunque estuvo presente en las reuniones, manifestó «delego en que hable Rocío (su mujer) que lo hace mejor que yo» (Reunión del 2/12/2011).

En los datos expuestos se ha evidenciado que las preguntas y cuestiones hechas a los padres sobre el contenido de la historia que narraban les han ayudado a indagar y pensar más sobre el evento o realidad de su país que estaba describiendo o mostrando visualmente. Según manifestaron, esos interrogantes les llevaron a «dejar de ver claro lo que siempre habían hecho» (Mar, 27/04/2012). Al tener que responder y decir el porqué de sus acciones o decisiones, comprobaron que no siempre poseían argumentos que justificasen el porqué de sus elecciones. En este sentido, las cuestiones formuladas a un narrador le suscitaron un extrañamiento que le llevó a nuevas indagaciones e, inevitablemente, a una mejor comprensión del contenido de la historia que estaba contando.

2) La influencia del director del colegio y de la tutora del grupo de alumnos ha sido fundamental en la participación de los padres en el desarrollo de este estudio. El director, aunque con una presencia no continua, dio legitimidad institucional a las sesiones celebradas; por su parte, la profesora proporcionó fundamento educativo a las acciones desarroIladas, y fue vía o puente facilitador de la comunicación entre los padres y la vida de sus hijos en el colegio, porque como dijo Eliot «... poder hablar con la maestra cuando ella no era maestra pero seguía estando en clase...» (Reunión, 25/05/2012).

3) La participación y experiencias de los padres y madres en este estudio han sido variadas aunque la proporción de los que han mantenido una continuidad y han desencadenado una base afectiva es de 4/7 de los que se comprometieron en la primera reunión a asistir. Un hecho evidente fue el contraste entre el conocimiento superficial, sin vínculos de amistad, que manifestaron tener unos padres de otros al principio del año escolar, y la relación más estrecha que existió entre ellos al final proporcionada por las vivencias en común de las creencias, sentimientos y 
emociones que justificaban las intenciones e interpretaciones que hicieron en las reuniones de narración. Estas sesiones les llevaron a entender cómo eran sus formas y estilos de vida.

A modo de reflexión sobre el futuro de las relaciones entre estas familias inmigrantes y el colegio Jaime Vera de Madrid al que asisten sus hijos e hijas, pensamos que este conocimiento fraguado entre ellas será una nueva motivación para que estos padres y madres vayan al colegio en el futuro, no solo para ver a la tutora sino, también, para verse y preguntarse cómo se encuentran.

\section{Referencias bibliográficas}

Banks, J.A. (2004). Diversity and citizenship education. San Francisco. Jossey-Bass.

Bolivar, A. (2006). Familia y Escuela: dos mundos Ilamados a trabajar en común. Revista de Educación, 339, 119-146.

Cabello, B. y Gradaílle, R. (2008). La educación social como práctica mediadora en las relaciones escuela-comunidad local. Revista Interuniversitaria de Pedagogía Social, $15,45-55$.

Castelli, S.; Mendel, M. \& Ravn, B. (Eds.) (2003). School, family, and community partnership in a world of differences and changes. Gdansk. University of Gdansk.

Chamberlin-Quinlisk, C. (2010). Cooperative learning as method and model in secondlanguage teacher education. Intercultural Education, vol. 21/3, 243-255.

Clandinin, D.J. y Connelly, F.M. (2000). Narrative Inquiry: Experience and Story in Qualitative Research. San Francisco, CA. Jossey-Bass.

Collet, J. y Tort, A. (2008). Espacios de participación». Cuadernos de Pedagogía, 78, 57-60.

Consejo de Europa (2007. Decisión 2006/1982/CE del Parlamento Europeo y del Consejo, de 18 de julio de 2006. Madrid. Ministerio de Ciencia y Tecnología.

Delgado, P. (2008). A Crianza Escola-Familia. Revista Interuniversitaria de Pedagogía Social, 15, 113-122.

Diez, E.-J.; y Terrón, E. (2006). Romper las barreras entre la familia y la escuela. Experiencia de investigación-acción en los centros escolares para promover la relación con las familias. Cultura y Educación, 18/3-4, 283-294.

Essomba, M.A. (2006). Liderar escuelas interculturales e inclusivas. Equipos directivos y profesorado ante la diversidad cultural y la inmigración. Barcelona. Graó.

Fernández Enguita, M. (1993). La profesión docente y la comunidad escolar: crónica de un desencuentro. Madrid. Editorial Morata.

Flick, U. (2012). Introducción a la investigación cualitativa. Madrid. Morata.

Franzé, A. (2008). Diversidad cultural en la escuela. Algunas contribuciones antropológicas». Revista de Educación, 345, 111-132.

García Castaño, F.; Rubio, M.; y Bouachra, O. (2008). Población inmigrante y escuela en España: un balance de investigación . Revista de Educación, 345, 23-60. 
Gibbs, G. (2012). El análisis de datos cualitativos en Investigación Cualitativa. Madrid. Morata.

Henderson, A.T.; Mapp, K.L., Johnson, V.R. \& Davis, D. (2007). Beyond the bake sale. The essential Guide to Family-School Partnerships. New York. The New Press.

Instituto Nacional de Estadística (2010). Informe anual. Madrid. Ministerio de Administraciones Públicas.

Johansson, G. (2009). Parental involvement in the development of a culture-based school curriculum. Intercultural Education, 20/4, 311-319.

Martínez, R.A.; Rodríguez, B.; y Gimeno, J.L. (2010). Áreas de cooperación entre los centros docentes y las familias. Estudio de caso. Educatio Siglo XXI, vol. 28, nº 1, pp. 127-156.

Mccaleb, S. (1997). Building communities of learners: a collaboration beween teachers, students, families, and communities. Mahwah, HJ. Lawrence Erlbaum Associates.

McKernan, J. (2008). Investigación-acción y curriculum. Madrid. Morata.

Ogbu, J. (2001). Understanding Cultural Diversity and Learnig, en J. Banks (Ed.), Handbook of Research on Multicultural Education. San Francisco: Jossey-Bass publishers, 582-583

Parcerisa, A. (2008). Educación Social en y con la institución escolar. Revista Interuniversitaria de Pedagogía Social, 15, 15-27.

Parker, W.C. (2003). Teaching democracy: Unity and diversity in public life. New York. Teachers College Press.

Torres, J. (2010). La justicia curricular. Madrid. Morata. 